\title{
Synthesis and Pharmacological Activities of Pyrano[2,3-d]pyrimidine and Pyrano[2,3-d]pyrimidine-5-one Derivatives as New Fused Heterocyclic Systems
}

\author{
Naglaa F. H. Mahmoud, Eman A. El-Bordany, and Galal A. Elsayed \\ Chemistry Department, Faculty of Science, Ain Shams University, Abbassia, Cairo 11566, Egypt \\ Correspondence should be addressed to Naglaa F. H. Mahmoud; naglaa.fawzy@yahoo.com
}

Received 7 April 2017; Revised 28 May 2017; Accepted 8 June 2017; Published 17 July 2017

Academic Editor: Orazio Nicolotti

Copyright (C) 2017 Naglaa F. H. Mahmoud et al. This is an open access article distributed under the Creative Commons Attribution License, which permits unrestricted use, distribution, and reproduction in any medium, provided the original work is properly cited.

\begin{abstract}
Various fused oxazine such as 4-(4-methoxyphenyl)-3,7-dimethyl-1,4-dihydro-5H-pyrazolo $\left[4^{\prime}, 3^{\prime}: 5,6\right]$ pyrano[2,3-d] [1,3] oxazin5-one 2 has been prepared and utilized as a starting material for novel pyrazolopyranopyrimidinones 3, 5, 6, and 7a-c and pyrazolopyranopyrimidines $4,9,10$, and 11 which are expected to possess considerable chemical and pharmacological activities. The structures of the new compounds have been elucidated by spectroscopic data and elemental analysis. The antioxidant and anticancer activities of synthesized products have been evaluated.
\end{abstract}

\section{Introduction}

Heterocyclic compounds are one of the most important molecules in the synthesis of pharmacologically active compounds [1-4]. Enaminonitriles have been utilized as starting materials for synthesis of huge number of heterocyclic compounds containing pyranopyrimidine [5-9] and pyridopyrimidine [10-13] moieties. Oxazines [14] and quinazolines [15] are natural products which exhibit a widespread spectrum of biological activity including antitumor [16-18], antiinflammatory [19], and antiplatelet [20] properties. In view of these reports and as a continuation of our previous work on heterocyclic chemistry [21-27], we aimed to incorporate the pyrano moiety into the 1,5-position of oxazinone and pyrimidinone ring systems to obtain new heterocyclic system which is anticipated to possess notable chemical and pharmacological activities. So we utilized enaminonitrile in the synthesis of pyrano[2,3-d][1,3] oxazin-5-one derivative and pyrano[2,3-d]pyrimidine and pyrano[2,3-d]pyrimidine5 -one derivatives in addition to studying their antioxidant activities.

\section{Results and Discussion}

2.1. Chemistry. Our present investigation aimed to synthesize of 6-amino-4-(4-methoxyphenyl)-3-methyl-1,4-dihydropyrano[2,3-c] pyrazole-5-carbonitrile $\mathbf{1}$ [28], which was used as a precursor for the synthesis of previously unreported 4-(4-methoxyphenyl)-3,7-dimethyl-1,4-dihydro-5Hpyrazolo[ $\left[4^{\prime}, 3^{\prime}: 5,6\right]$ pyrano[2,3-d][1,3] oxazin-5-one 2 as new heterocyclic compound containing oxazine and pyranopyrazole moieties. Compound $\mathbf{1}$ has been prepared by fourcomponent one-pot reaction [29] and three-component onepot reaction [30] (Scheme 1).

Treatment of $\mathbf{1}$ by freshly distilled acetic anhydride afforded $\mathbf{2}$ as a sole product. The structure of $\mathbf{2}$ was elucidated by spectral analysis. The IR spectrum showed a strong absorption band at $1732 \mathrm{~cm}^{-1}$ corresponding to lactonic carbonyl group and the disappearance of the cyano group. Also the structure was confirmed by elemental analysis; ${ }^{1} \mathrm{H}-\mathrm{NMR}$ spectrum showed a singlet at $\delta 12.52 \mathrm{ppm}$ of NH, a doublet at $\delta 7.162-7.136 \mathrm{ppm}$ and $\delta 6.84-6.81 \mathrm{ppm}$ of aromatic protons, a singlet at $\delta 4.7 \mathrm{ppm}$ of benzylic proton, and a singlet at $\delta 3.68 \mathrm{ppm}$ of three protons for $\mathrm{OCH}_{3}$ group. 


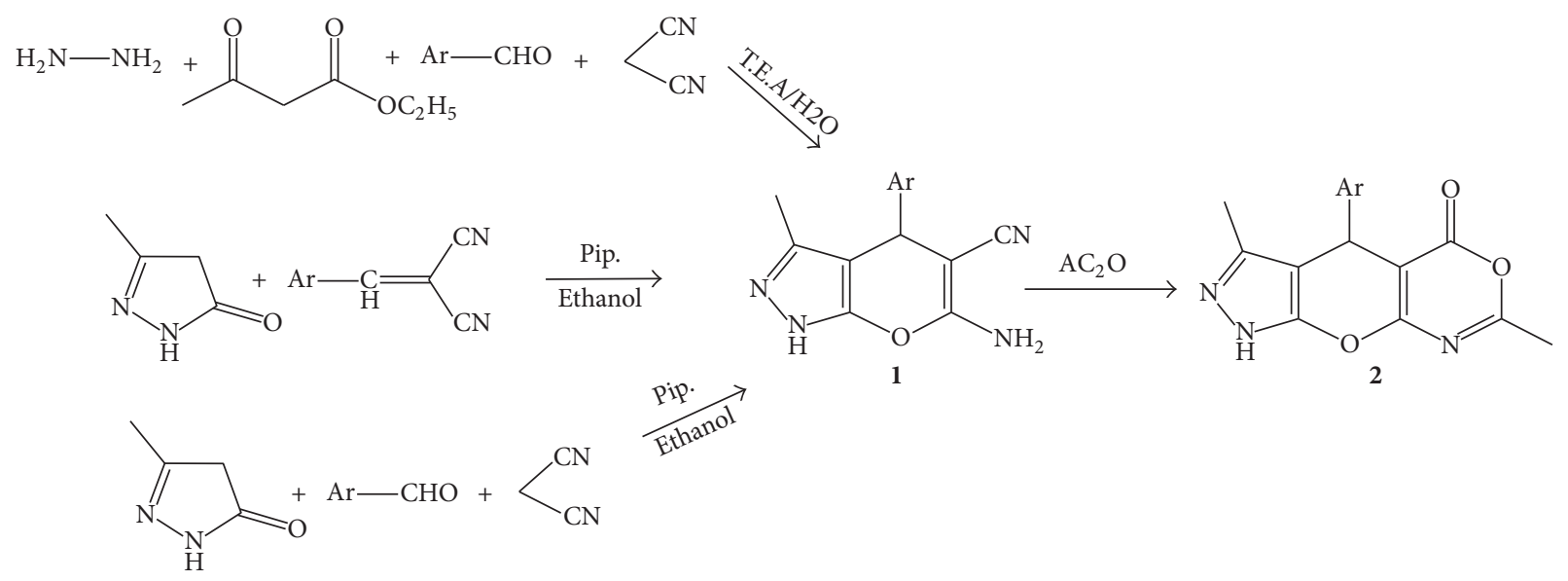

Ammonolysis of $\mathbf{2}$ by fusion with ammonium acetate and/or formamide afforded 4-(4-methoxyphenyl)-3,7-dimethyl4,6-dihydro-pyrazolo $\left[4^{\prime}, 3^{\prime}: 5,6\right]$ pyrano[2,3-d]pyrimidine$5(1 \mathrm{H})$-one 3 . The structure of 3 was confirmed by the IR spectrum which exhibited strong absorption bands at $3200,3281,1664$, and $1610 \mathrm{~cm}^{-1}$ corresponding to $v_{\mathrm{NH}}, v_{\mathrm{C}=\mathrm{O}}$, and $v_{\mathrm{C}=\mathrm{N}}$, respectively and lacked a band corresponding to lactonic carbonyl group and also the presence of acidic hydrogen in the ${ }^{1} \mathrm{H}-\mathrm{NMR}$ spectrum at $\delta$ 12.38. The electrophilicity of the lactonic carbonyl group of compound $\mathbf{2}$ was studied by nucleophilic reaction with 1,2-diaminobenzene and thiosemicarbazide, as 1,4dinucleophile, to afford 4-(4-methoxyphenyl)-3,7-dimethyl4-hydrobenzimidazolo[1,2,c]pyrazolo[ $\left[4^{\prime} 3^{\prime}: 5,5\right]$ pyrano[2,3d]pyrimidine 4 and N-[4-(4-methoxyphenyl)-3,7-dimethyl5-oxo-1,4-dihydro-pyrazolo $\left[4^{\prime}, 3^{\prime}: 5,6\right]$ pyrano[2,3-d]pyrimidin-6(5H)-yl]thiourea 5 , as sole products. The pyrazolopyranopyrimidine derivative $\mathbf{4}$ was formed via ring opening of the oxazine ring by an amino group followed by ring closer and then dehydration with the other amino group. The IR spectrum of compound $\mathbf{4}$ showed strong absorption bands at 3180 and $1609 \mathrm{~cm}^{-1}$ corresponding to $\nu_{\mathrm{NH}}$ and $\nu_{\mathrm{C}=\mathrm{N}}$. The IR spectrum of compound 5 showed well defined absorption bands at 3251, 3158, 3121, 1665, 1619, and $1254 \mathrm{~cm}^{-1}$ corresponding to $\nu_{\mathrm{NH} 2}, \nu_{\mathrm{NH}}, v_{\mathrm{C}=\mathrm{O}}, \nu_{\mathrm{C}=\mathrm{N}}$, and $\nu_{\mathrm{C}=\mathrm{S}}$. Compound 2 was allowed to react with primary amines, namely, hydrazine hydrate, 4-methylaniline, 4-methoxyaniline, and/ or 4-aminoaniline, to afford the pyrazolopyranopyrimidine derivatives 6 and $7 \mathbf{a}-\mathbf{c}$, respectively (Scheme 2 ).

4-(4-Methoxyphenyl)-3,7dimethy-1-4,6-dihydropyrazolo $\left[4^{\prime}, 3^{\prime}: 5,6\right]$ pyrano $[2,3-\mathrm{d}]$ pyrimidin-5 $(1 \mathrm{H})$-thione 8 was synthesized by sulfurization of $\mathbf{3}$ using Lawson's reagent or by reaction of thiourea with 5-chloro-4-(4-methoxyphenyl)-3,7-dimethyl-1,4-dihydropy-razolo $\left[4^{\prime}, 3^{\prime}: 5,6\right]$ pyrano[2,3-d] pyrimidine 9. Compound 9 was prepared by chlorination of 3 by $\mathrm{POCl}_{3}$ and $\mathrm{PCl}_{5}$ mixture. Hydrazinolysis of 9 afforded 5-hydrazino-4-(4-methoxyphenyl)-3,7-dimethyl-1,4-dihydropyrazolo $\left[4^{\prime}, 3^{\prime}: 5,6\right]$ pyrano $[2,3$-d $]$ pyrimidine 10.

IR spectrum of compound $\mathbf{1 0}$ showed strong absorption bands at $3215,3156,3161$, and $1612 \mathrm{~cm}^{-1}$ corresponding to $v_{\mathrm{NH} 2}, v_{\mathrm{NH}}$, and $v_{\mathrm{C}=\mathrm{N}}$. Also the structure was confirmed by ${ }^{1} \mathrm{H}$-NMR spectrum that showed a singlet at $\delta 12.01 \mathrm{ppm}$ of $\mathrm{NH}$, multiplet at $\delta 7.83 \mathrm{ppm}$ of aromatic protons, a singlet at $\delta 4.8 \mathrm{ppm}$ of benzylic proton, a singlet at $\delta 3.7 \mathrm{ppm}$ of three protons for $\mathrm{OCH}_{3}$ group, and a singlet at $\delta 5.354$ of $\mathrm{NH}_{2}$.

Acetylation of $\mathbf{1 0}$ by refluxing with acetic anhydride gave the diacetyl derivative $\mathbf{1 1}$ and not the expected triazolo derivative 12. The structure of $\mathbf{1 1}$ was confirmed by the spectral data such as the IR which showed the absorbance for the carbonyl group at 1732 and $1695 \mathrm{~cm}^{-1}$ and the two methyl groups signals at $\delta 2.678 \mathrm{ppm}$ for $2 \mathrm{CH}_{3}$ in ${ }^{1} \mathrm{H}-\mathrm{NMR}$ spectrum (Scheme 3).

2.2. Pharmacological Activity. Antioxidant activity using 2,2' -azinobis(3-ethylbenzothiazoline-6-sulfonic acid) (ABTS) inhibition. The prepared compounds were tested for antioxidant activity as reflected in their ability to inhibit oxidation in rat brain and kidney homogenates, from the result in Table 1. Compounds 1, 4, 7a, and 10 exhibit a similar antioxidant activity to ascorbic acid, used as standard. Compounds $\mathbf{8}$ and 11 exhibited moderate antioxidant activity, while 2, 3, 7b, 5, and 9 showed lower activity.

Bleomycin Dependent DNA Damage. The bleomycins are a family of glycopeptides antibiotics [31] that are routinely used as antitumor agents. The bleomycin assay has been adopted for assessing the prooxidant activity of food antioxidants. The antitumor antibiotic bleomycin binds iron ions and DNA. If the samples are able to reduce bleomycin- $\mathrm{Fe}^{3+}$ to bleomycin-Fe, DNA degradation in the system will be stimulated, resulting in a positive test for prooxidant activity. DNA 


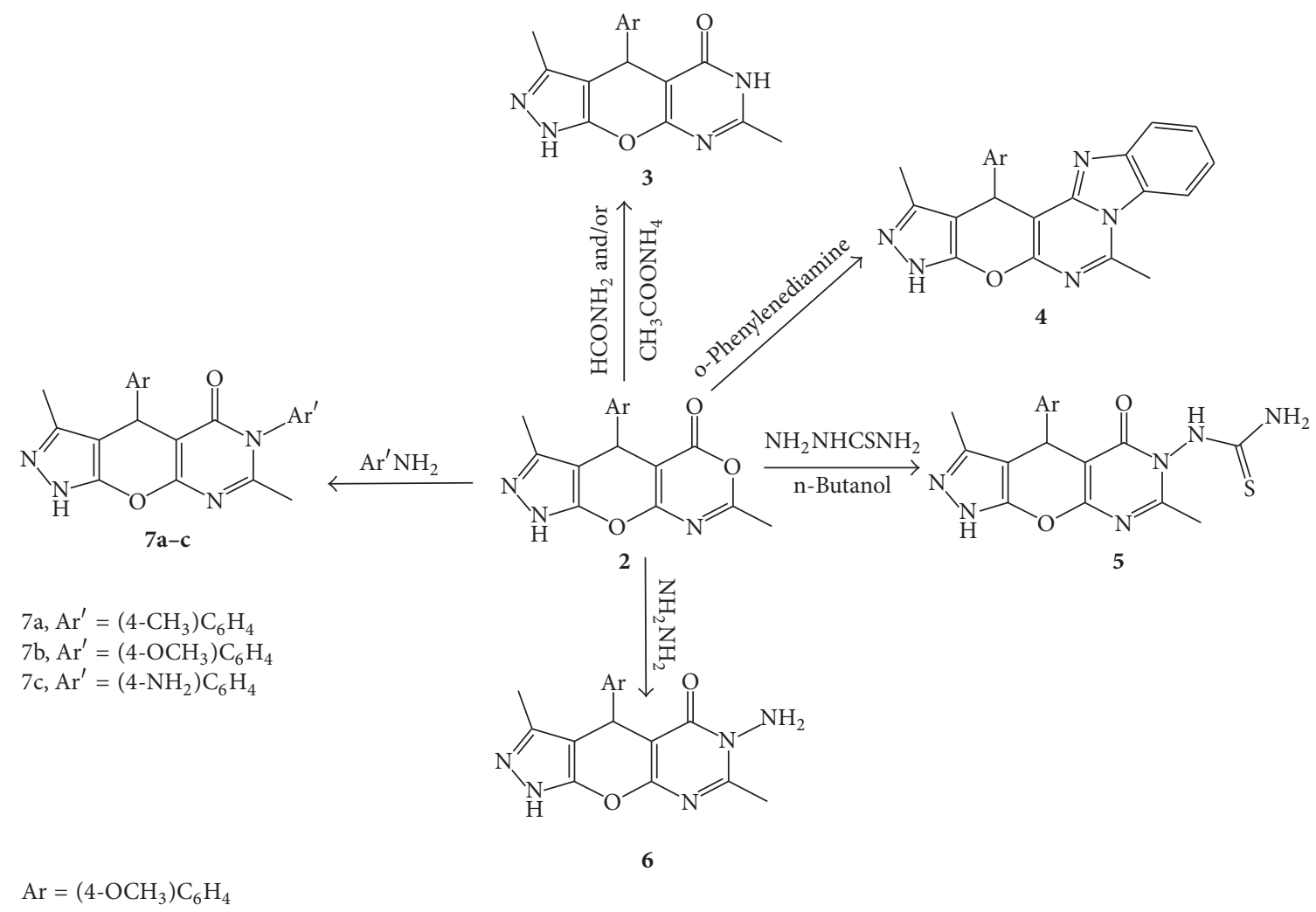

SCHEMe 2

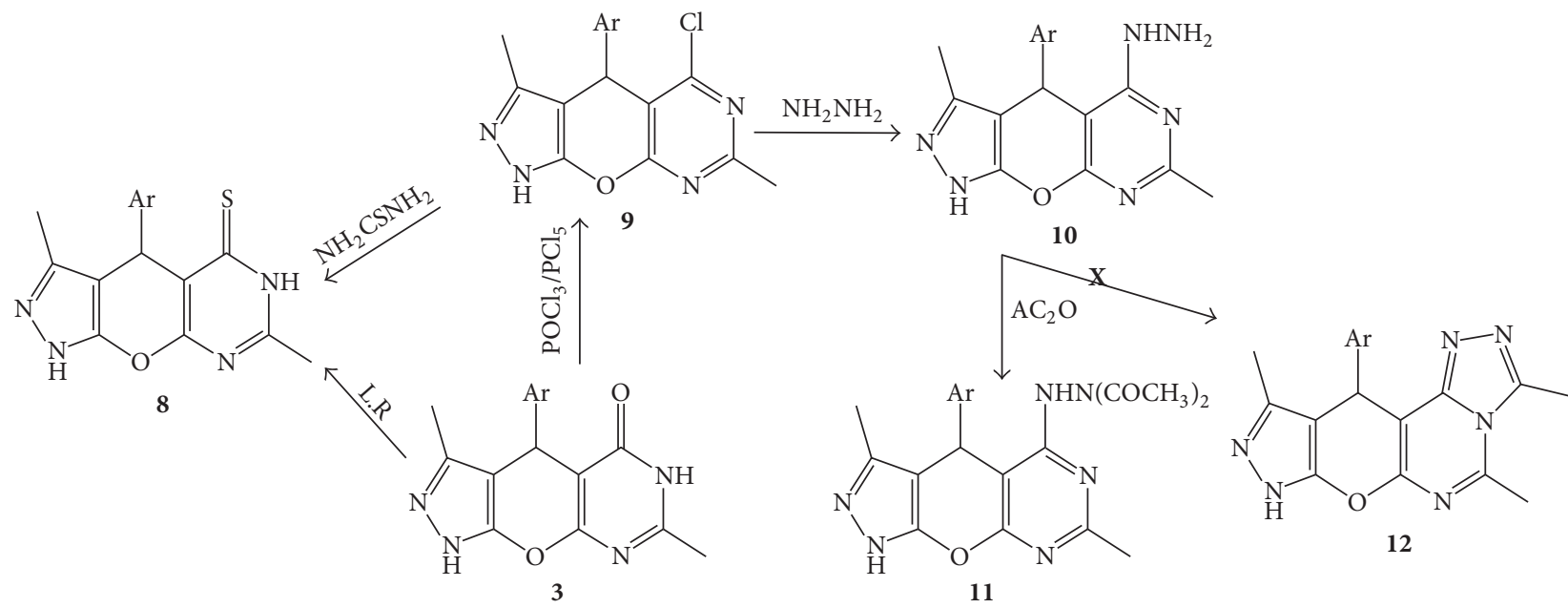

Scheme 3

degradation is accompanied by the formation of a product similar to malondialdehyde.

L-Ascorbic acid was used as the reducing agent to reduce $\mathrm{Fe}^{3+}$ to $\mathrm{Fe}^{2+}$. The synthesized compounds were selected for bleomycin dependent DNA damage testing (Table 1).

Results in Table 1 showed that compounds $1,4,7 \mathbf{a}, 8$, and $\mathbf{1 0}$ have the ability to protect DNA from the damage induced by bleomycin. On the other hand, the rest of the compounds exhibited weak activities.

Cytotoxic Activity of Some Compounds against Human Tumor Cells. The prepared compounds were tested for cytotoxic activity against four human tumor cells. Best results were observed for compounds $1,4,7 \mathrm{a}$, and 10 which were found 
TABLE 1: The antioxidant activities of the synthesized compounds.

\begin{tabular}{lccc}
\hline \multirow{2}{*}{ Compound number } & \multicolumn{2}{c}{ Antioxidant activity } & Bleomycin dependent DNA damage \\
\hline 1 & Absorbance & Inhibition \% & 0.098 \\
2 & 0.111 & 78.6 & 0.127 \\
3 & 0.427 & 17.9 & 0.135 \\
4 & 0.419 & 8.4 & 0.083 \\
5 & 0.062 & 24.4 & 0.138 \\
$7 \mathrm{a}$ & 0.393 & 84.0 & 0.091 \\
$7 \mathrm{~b}$ & 0.083 & 17.7 & 0.151 \\
8 & 0.428 & 47.3 & 0.089 \\
9 & 0.274 & 14.2 & 0.142 \\
10 & 0.446 & 88.5 & 0.066 \\
11 & 0.060 & 31.7 & 0.112 \\
Ascorbic acid & 0.355 & 89.2 & 0.076 \\
ABTS & 0.065 & - & - \\
\hline
\end{tabular}

TABLE 2: The cytotoxic activity of some compounds against human tumor cells.

\begin{tabular}{|c|c|c|c|c|}
\hline \multirow{2}{*}{ Compounds } & \multicolumn{4}{|c|}{ In vitro cytotoxicity IC50 $(\mu \mathrm{g} / \mathrm{ml})^{\circ}$} \\
\hline & HePG2 & HCT-116 & PC3 & MCF-7 \\
\hline $5-\mathrm{FU}$ & $7.9 \pm 0.12$ & $5.3 \pm 0.14$ & $8.3 \pm 0.25$ & $5.4 \pm 0.21$ \\
\hline 1 & $9.4 \pm 0.27$ & $13.5 \pm 1.65$ & $16.9 \pm 1.43$ & $26.8 \pm 1.52$ \\
\hline 2 & $82.1 \pm 5.34$ & $95.9 \pm 6.34$ & $>100$ & $>100$ \\
\hline 3 & $45.6 \pm 3.21$ & $51.1 \pm 3.50$ & $61.1 \pm 4.96$ & $37.2 \pm 2.07$ \\
\hline 4 & $8.4 \pm 0.30$ & $9.0 \pm 0.41$ & $16.0 \pm 1.30$ & $14.6 \pm 1.36$ \\
\hline 5 & $36.2 \pm 3.22$ & $52.2 \pm 3.67$ & $41.3 \pm 2.65$ & $40.1 \pm 3.22$ \\
\hline $7 a$ & $8.7 \pm 0.26$ & $13.61 \pm .48$ & $17.9 \pm 1.57$ & $19.5 \pm 1.81$ \\
\hline $7 b$ & $63.5 \pm 3.98$ & $>100$ & $85.2 \pm 6.31$ & $85.5 \pm 5.62$ \\
\hline 8 & $16.4 \pm 1.04$ & $31.4 \pm 2.64$ & $19.9 \pm 1.68$ & $26.9 \pm 1.91$ \\
\hline 9 & $52.0 \pm 2.83$ & $69.2 \pm 4.05$ & $47.2 \pm 2.54$ & $58.5 \pm 3.54$ \\
\hline 10 & $8.3 \pm 0.41$ & $11.2 \pm 0.86$ & $8.9 \pm 0.50$ & $9.1 \pm 0.65$ \\
\hline 11 & $22.1 \pm 1.56$ & $37.8 \pm 1.93$ & $26.4 \pm 1.79$ & $30.0 \pm 2.80$ \\
\hline
\end{tabular}

IC50 ( $\mu \mathrm{g} / \mathrm{ml}):$ 1-10 (very strong), 11-20 (strong), 21-50 (moderate), 51-100 (weak), and above 100 (noncytotoxic); 5-FU = 5-fluorouracil.

to very strong cytotoxic compounds. Compound $\mathbf{8}$ was also considered strongly cytotoxic. Finally, compounds 3, 5, and 11 were moderate in their cytotoxic activity and compounds $\mathbf{2}, \mathbf{7 b}$, and 9 were weakly cytotoxic (Table 2).

2.3. Experimental. All melting points were measured on a Gallenkamp electric melting point apparatus and are uncorrected. The infrared spectra were recorded in potassium bromide disks on a pyeUnicam SP-3-300 and Shimdazu FTIR 8101 PC infrared spectrophotometers.

The ${ }^{1} \mathrm{H}-\mathrm{NMR}$ and ${ }^{13} \mathrm{C}-\mathrm{NMR}$ were recorded at a Varian Mercury VX-300 MHz and $75 \mathrm{MHz}$, respectively, using TMS as internal standard in deuterated chloroform $\left(\mathrm{CDCl}_{3}\right)$ or deuterated dimethyl sulfoxide (DMSO-d6). Chemical shifts are measured in ppm. The mass spectra were recorded on a Shimadzu GCMS-QP-1000EX mass spectrometer at $70 \mathrm{eV}$. Elemental analyses were carried out at the Microanalytical Center of Cairo University. All the reactions and the purity of the new compounds were followed and checked by TLC using TLC aluminum sheets silica gel $\mathrm{F}_{254}$.

\subsection{Synthesis of 6-Amino-4-(4-methoxyphenyl)-3-methyl-1,4- dihydropyrano[2,3-c]pyrazole-5-carbonitrile (1)}

Method 1. An equimolar mixture of anisaldehyde $(10 \mathrm{mmol}$, $1.36 \mathrm{~mL})$, ethyl acetoacetate $(10 \mathrm{mmol}, 1.3 \mathrm{~mL})$, hydrazine hydrate $(0.5 \mathrm{~mL})$, and malononitrile $(10 \mathrm{mmol}, 0.66 \mathrm{~g})$ was stirred for $30 \mathrm{~min}$ at room temperature in the presence of triethyl amine and water $(5 \mathrm{ml})$. The precipitate was filtered off and recrystallized from ethanol, yield (81\%).

Method 2. A mixture of 3-methyl-5-pyrazolone (10 mmol, $0.98 \mathrm{~g})$ and arylidine $(10 \mathrm{mmol}, 1.1 \mathrm{~g})$ was refluxed in $(30 \mathrm{~mL})$ ethanol and 5 drops of piperidine for $2 \mathrm{~h}$. The precipitate was filtrated off and recrystallized from ethanol to give solid 1, yield (87\%). 
Method 3. A mixture of 3-methyl-5-pyrazolone (10 mmol, $0.98 \mathrm{~g})$, anisaldehyde (10 mmol, $1.36 \mathrm{~mL})$, and malononitrile $(10 \mathrm{mmol}, 0.66 \mathrm{~g})$ was refluxed in $(30 \mathrm{~mL})$ ethanol in presence of 5 drops of piperidine for $2 \mathrm{~h}$. The precipitate was filtrated off and recrystallized from ethanol to give solid 1, yield (80\%)

2.5. Synthesis of 4-(4-Methoxyphenyl)-3,7-dimethyl-1,4dihydro-5H-pyrazolo $\left[4^{\prime}, 3^{\prime}: 5,6\right]$ pyrano $[2,3-d][1,3]$ oxazin-5one (2). A mixture of $\mathbf{1}(10 \mathrm{mmol}, 2.82 \mathrm{~g})$ and freshly distilled acetic anhydride $(20 \mathrm{~mL})$ was heated under reflux for $3 \mathrm{~h}$. The solid that deposited after distilling the excess solvent was collected and recrystallized from ethanol to give (2).

m.p. over $300^{\circ} \mathrm{C}$. Anal. Calcd. for $\mathrm{C}_{17} \mathrm{H}_{15} \mathrm{~N}_{3} \mathrm{O}_{4}$ (325.32): C, 62.76; H, 4.65; N, 12.92. Found: C, 63.07; H, 4.29; N, 13.10. FTIR (KBr) $\left(\mathrm{cm}^{-1}\right): 3157(\mathrm{NH}), 1732(\mathrm{C}=\mathrm{O}), 1624(\mathrm{C}=\mathrm{N})$, $1606(\mathrm{C}=\mathrm{C}) .{ }^{1} \mathrm{H}-\mathrm{NMR}\left(\mathrm{DMSO}-d_{6}\right): \delta_{\mathrm{H}}(\mathrm{ppm}) 12.52(\mathrm{~s}, 1 \mathrm{H}$, $\mathrm{NH}, \mathrm{D}_{2} \mathrm{O}$ exchangeable), $7.162-7.136(\mathrm{~d}, J=7.8,2 \mathrm{H}, \mathrm{Ar}-\mathrm{H})$, 6.84-6.81 (d, $J=9, \mathrm{H}, \mathrm{Ar}-\mathrm{H}), 4.7$ ( $\mathrm{s}, 1 \mathrm{H}$, benzylic proton), $3.68\left(\mathrm{~s}, 3 \mathrm{H}, \mathrm{OCH}_{3}\right), 2,16\left(\mathrm{~s}, 3 \mathrm{H}, \mathrm{CH}_{3}\right)$ and $1.831\left(\mathrm{~s}, 3 \mathrm{H}, \mathrm{CH}_{3}\right)$. ${ }^{13} \mathrm{C}-\mathrm{NMR}\left(75 \mathrm{MHz}\right.$, DMSO- $\left.d_{6}\right): \delta 173.7,165,163.4,161.9,157.2$, 138.6, 134.2, 130.3, 130.3, 114.4, 114.5, 112.9, 99.8, 55.5, 34.4, 15.7, 13.4. MS, $m / z(\%): 325\left(\mathrm{M}^{\bullet+}, 6\right), 281(27), 241(13), 218(100)$, 107 (28).

2.6. Synthesis of 4-(4-Methoxyphenyl)-3,7-dimethyl-4,6-dihydropyrazolo $\left[4^{\prime}, 3^{\prime}: 5,6\right]$ pyrano $[2,3-d]$ pyrimidine-5-(1H)-one (3). A mixture of $2(10 \mathrm{mmol}, 3.25 \mathrm{~g})$ and formamide $(10 \mathrm{~mL})$ or excess ammonium acetate was refluxed for $4 \mathrm{~h}$. After cooling white solid was collected, washed with water, dried, and recrystallized from benzene: ethanol mixture $(1: 1)$ to give $(3)$.

m.p. over $300^{\circ} \mathrm{C}$ Anal. Calcd. for $\mathrm{C}_{17} \mathrm{H}_{16} \mathrm{~N}_{4} \mathrm{O}_{3}$ (324.33): C, 62.95; H, 4.97; N, 17.27. Found: C, 62.07; H, 4.92; N, 17.10. FTIR (KBr) $\left(\mathrm{cm}^{-1}\right): 3200,3281(\mathrm{NH}), 1664(\mathrm{C}=\mathrm{O}), 1610$ $(\mathrm{C}=\mathrm{N}){ }^{1} \mathrm{H}-\mathrm{NMR}\left(\mathrm{DMSO}-d_{6}\right): \delta_{\mathrm{H}}(\mathrm{ppm}) 12.381(\mathrm{~s}, 1 \mathrm{H}, \mathrm{NH}$, $\mathrm{D}_{2} \mathrm{O}$ exchangeable), $12.01\left(\mathrm{~s}, 1 \mathrm{H}, \mathrm{NH}, \mathrm{D}_{2} \mathrm{O}\right.$ exchangeable), $7.359-7.334(\mathrm{~d}, J=7.5,2 \mathrm{H}, \operatorname{Ar}-\mathrm{H}), 6.790-6.762(\mathrm{~d}, J=$ 8.4, 2H, Ar-H), 4.899 (s, $1 \mathrm{H}$, benzylic proton), 3.668 (s, 3H, $\left.\mathrm{OCH}_{3}\right), 2.176\left(\mathrm{~s}, 3 \mathrm{H}, \mathrm{CH}_{3}\right)$ and $1.791\left(\mathrm{~s}, 3 \mathrm{H}, \mathrm{CH}_{3}\right) . \mathrm{MS}, \mathrm{m} / z$ (\%): $324\left(\mathrm{M}^{\bullet+}, 16\right), 281$ (19), 240 (24), 217 (100), 107 (51).

2.7. Synthesis of 4-(4-Methoxyphenyl)-3,7-dimethyl-4-hydrobenzoimidazolo[1,2-c]pyrazolo $\left[4^{\prime}, 3^{\prime}: 5,5\right]$ pyrano $[3,2-d]$ pyrimidine (4). A mixture of 2 (10 mmol, $3.25 \mathrm{~g})$ and 2-amino aniline $(10 \mathrm{mmol}, 1.08 \mathrm{~g})$ in n-butanol $(20 \mathrm{~mL})$ was heated under reflux for $4 \mathrm{~h}$. The solid that separated after concentration and cooling was filtered off and recrystallized from petroleum ether at $60-80^{\circ} \mathrm{C}$.

m.p. 102-104 ${ }^{\circ} \mathrm{C}$ Anal. Calcd. For $\mathrm{C}_{23} \mathrm{H}_{19} \mathrm{~N}_{5} \mathrm{O}_{2}$ (397): C, 69.51; H, 4.82; N, 17.62. Found: C, 69.26; H, 4.78; N, 17.23. FTIR $(\mathrm{KBr})\left(\mathrm{cm}^{-1}\right): 3180(\mathrm{NH}), 1609(\mathrm{C}=\mathrm{N}){ }^{1} \mathrm{H}-\mathrm{NMR}\left(\mathrm{DMSO}-d_{6}\right)$ : $\delta_{\mathrm{H}}(\mathrm{ppm}) 11.252\left(\mathrm{~s}, 1 \mathrm{H}, \mathrm{NH}, \mathrm{D}_{2} \mathrm{O}\right.$ exchangeable $), 8.023-7.215$ ( $\mathrm{m}, 8 \mathrm{H}, \mathrm{Ar}-\mathrm{H}), 5.099$ (s, $1 \mathrm{H}$, benzylic proton), 3.212 (s, $3 \mathrm{H}$, $\left.\mathrm{OCH}_{3}\right), 2.801\left(\mathrm{~s}, 3 \mathrm{H}, \mathrm{CH}_{3}\right)$ and 2,176 (s, 3H, $\left.\mathrm{CH}_{3}\right) . \mathrm{MS}, \mathrm{m} / z$ (\%): $395\left(\mathrm{M}^{\bullet+}, 7\right), 317$ (9), 290 (12), 267 (10), 181 (25), 80 (100).

2.8. Synthesis of $\mathrm{N}$-[4-(4-Methoxyphenyl)-3,7-dimethyl-5oxo-1,4-dihydropyrazolo $\left[4^{\prime}, 3^{\prime}: 5,6\right]$ pyrano $[2,3-d]$ pyrimidine$6(5 \mathrm{H})$-yl]thiourea (5). A mixture of $2(10 \mathrm{mmol}, 3.25 \mathrm{~g})$ and thiosemicarbazide (10 mmol, $0.91 \mathrm{~g}$ ) in dimethylformamide
$(20 \mathrm{~mL})$ was heated under reflux for $6 \mathrm{~h}$. The solid that was separated after concentration and cooling was filtered off, washed with water, and recrystallized from ethanol to give (5).

m.p. 265-267 ${ }^{\circ} \mathrm{C}$ Anal. Calcd. for $\mathrm{C}_{18} \mathrm{H}_{18} \mathrm{~N}_{6} \mathrm{O}_{3} \mathrm{~S}$ (398.44): C, 54.26; H, 4.55; N, 21.09; S, 8.05. Found: C, 53.70; H, 4.19; $\mathrm{N}, 20.83$. FTIR $(\mathrm{KBr})\left(\mathrm{cm}^{-1}\right): 3251,3158,3121\left(\mathrm{NH}_{2}, \mathrm{NH}\right), 1665$ (CO), $1619(\mathrm{C}=\mathrm{N})$ and $1254(\mathrm{C}=\mathrm{S}) .{ }^{1} \mathrm{H}-\mathrm{NMR}\left(\mathrm{DMSO}-d_{6}\right): \delta_{\mathrm{H}}$ (ppm) 11.912 (s, 1H, NH, $\mathrm{D}_{2} \mathrm{O}$ exchangeable), 7.923-7.751 (m, $4 \mathrm{H}, \mathrm{Ar}-\mathrm{H}), 8.213$ (s, $1 \mathrm{H}, \mathrm{NH}, \mathrm{D}_{2} \mathrm{O}$ exchangeable), 6.754 (s, 2H, $\mathrm{NH}_{2}, \mathrm{D}_{2} \mathrm{O}$ exchangeable), 5.013 (s, $1 \mathrm{H}$, benzylic proton), 3.934 $\left(\mathrm{s}, 3 \mathrm{H}, \mathrm{OCH}_{3}\right), 2.178\left(\mathrm{~s}, 3 \mathrm{H}, \mathrm{CH}_{3}\right)$ and $1.987\left(\mathrm{~s}, 3 \mathrm{H}, \mathrm{CH}_{3}\right) .{ }^{13} \mathrm{C}-$ NMR $\left(75 \mathrm{MHz}, \mathrm{DMSO}-d_{6}\right): \delta 182.7,163.2,161.9,160.3,158.5$, $157.1,139.8,135.2,130.3,130.3,114.6,114.6,113.8,102.9,55.9$, 35.7, 22.3, 13.3,. MS, $m / z(\%): 398\left(\mathrm{M}^{\bullet+}, 23\right), 323(20), 291(18)$, 107 (100), 75 (21).

2.9. Synthesis of 6-Amino-4-(4-methoxyphenyl)-3,7-dimethyl4,6-dihydropyrazolo $\left[4^{\prime}, 3^{\prime}: 5,6\right]$ pyrano $[2,3-d]$ pyrimidin-5(1H)one (6). To a solution of 2 (10 mmol, $3.25 \mathrm{~g})$ in $20 \mathrm{~mL}$ of ethanol, hydrazine hydrate $(0.5 \mathrm{~mL})$ was added. The reaction mixture was refluxed for $4 \mathrm{~h}$. The separated solid was filtered, dried, and recrystallized from ethanol to give (6).

m.p. 268-290 ${ }^{\circ} \mathrm{C}$ Anal. Calcd. for $\mathrm{C}_{17} \mathrm{H}_{17} \mathrm{~N}_{5} \mathrm{O}_{3}$ (339.35): C, 60.17; H, 5.05; N, 20.64. Found: C, 60.07; H, 4.94; N, 20.53. FTIR (KBr) $\left(\mathrm{cm}^{-1}\right): 3325,3210,3154\left(\mathrm{NH}_{2}, \mathrm{NH}\right), 1669$ (CO), $1609(\mathrm{C}=\mathrm{N}) .{ }^{1} \mathrm{H}-\mathrm{NMR}\left(\mathrm{DMSO}-d_{6}\right): \delta_{\mathrm{H}}(\mathrm{ppm}) 12.012(\mathrm{~s}, 1 \mathrm{H}$, $\mathrm{NH}, \mathrm{D}_{2} \mathrm{O}$ exchangeable), 7.632-7.115 (m, 4H, Ar-H), 6.845 (s, $2 \mathrm{H}, \mathrm{NH}_{2}, \mathrm{D}_{2} \mathrm{O}$ exchangeable), 5.013 (s, $1 \mathrm{H}$, benzylic proton), $3.798\left(\mathrm{~s}, 3 \mathrm{H}, \mathrm{OCH}_{3}\right), 2.867\left(\mathrm{~s}, 3 \mathrm{H}, \mathrm{CH}_{3}\right)$ and $1.872(\mathrm{~s}, 3 \mathrm{H}$, $\left.\mathrm{CH}_{3}\right)$. MS, $m / z(\%): 322\left(\mathrm{M}^{\bullet+}-\mathrm{NH}_{3}, 23\right), 282(19), 240(10)$, $80(100)$.

2.10. General Procedure for the Synthesis of Pyrazolopyranopyrimidine $(7 \boldsymbol{a}-\boldsymbol{c})$. A solution of $2(10 \mathrm{mmol}, 3.25 \mathrm{~g})$ in dimethylformamide $(20 \mathrm{~mL})$ and $(10 \mathrm{mmol})$ of 4 -methyl aniline, 4-methoxyaniline, and/or 4-amino aniline was heated under reflux for $5 \mathrm{~h}$. The solid that was separated out after concentration and cooling was recrystallized from the proper solvent to give $(7 \mathbf{a}-\mathbf{c})$.

2.11. 4-(4-Methoxyphenyl)-3,7-dimethyl-6-(p-tolyl)-4,6-dihydropyrazolo $\left[4^{\prime}, 3^{\prime}: 5,6\right]$ pyrano $[2,3-d]$ pyrimidin-5(1H)-one (7a). Solvent: ethanol m.p. $278-280^{\circ}$ C. Anal. Calcd. for $\mathrm{C}_{24} \mathrm{H}_{22} \mathrm{~N}_{4} \mathrm{O}_{3}$ (414.46): C, 69.55; H, 5.35; N, 13.52. Found: C, 68.67; H, 4.89; N, 12.98. FTIR (KBr) $\left(\mathrm{cm}^{-1}\right): 3191(\mathrm{NH}), 1682$ (CO), $1608(\mathrm{C}=\mathrm{N}) .{ }^{1} \mathrm{H}-\mathrm{NMR}\left(\mathrm{DMSO}-d_{6}\right): \delta_{\mathrm{H}}(\mathrm{ppm}) 10.972$ (s, 1H, NH, $\mathrm{D}_{2} \mathrm{O}$ exchangeable), 7.963-7.785 (m, 8H, Ar-H), $4.876\left(\mathrm{~s}, 1 \mathrm{H}\right.$, benzylic proton), $3.912\left(\mathrm{~s}, 3 \mathrm{H}, \mathrm{OCH}_{3}\right), 2.911(\mathrm{~s}$, $\left.3 \mathrm{H}, \mathrm{CH}_{3}\right)$ and $2.176\left(\mathrm{~s}, 3 \mathrm{H}, \mathrm{CH}_{3}\right)$ and $1.987\left(\mathrm{~s}, 3 \mathrm{H}, \mathrm{CH}_{3}\right) . \mathrm{MS}$, $m / z(\%): 414\left(\mathrm{M}^{\bullet+}, 17\right), 386(8), 307$ (19), 107 (100).

2.12. 4,6-Bis(4-methoxyphenyl)-3,7-dimethyl-4,6-dihydropyrazolo $\left[4^{\prime}, 3^{\prime}: 5,6\right]$ pyrano $[2,3-d]$ pyrimidin-5(1H)-one $(7 \boldsymbol{b})$. Solvent: ethanol. m.p. over $300^{\circ} \mathrm{C}$ Anal. C; alcd. for $\mathrm{C}_{24} \mathrm{H}_{22} \mathrm{~N}_{4} \mathrm{O}_{4}$ (430.46): C, 66.97; H, 5.15; N, 13.02. Found: C, 66.17; H, 4.98; N, 13.41 FTIR (KBr) $\left(\mathrm{cm}^{-1}\right): 3154(\mathrm{NH}), 1685$ (CO), $1611(\mathrm{C}=\mathrm{N}) .{ }^{1} \mathrm{H}-\mathrm{NMR}\left(\mathrm{DMSO}-d_{6}\right): \delta_{\mathrm{H}}(\mathrm{ppm}) 11.241$ 
(s, 1H, NH, $\mathrm{D}_{2} \mathrm{O}$ exchangeable), 8.054-7.815 (m, 8H, Ar-H), 5.045 ( $\mathrm{s}, 1 \mathrm{H}$, benzylic proton), $3.912\left(\mathrm{~s}, 6 \mathrm{H}, 2 \mathrm{OCH}_{3}\right), 2.832$ $\left(\mathrm{s}, 3 \mathrm{H}, \mathrm{CH}_{3}\right), 2.092\left(\mathrm{~s}, 3 \mathrm{H}, \mathrm{CH}_{3}\right)$ and $1.898\left(\mathrm{~s}, 3 \mathrm{H}, \mathrm{CH}_{3}\right) . \mathrm{MS}$, m/z (\%): $430\left(\mathrm{M}^{\circ+}, 8\right), 402$ (7), 295 (19), 80 (100).

2.13. 6-(4-Aminophenyl)-4-(methoxyphenyl)-3,7-dimethyl4,6-dihydropyrazolo $\left[4^{\prime}, 3^{\prime}: 5,6\right]$ pyrano $[2,3-d]$ pyrimidin-5(1H)one (7c). Solvent: dioxane. m.p. over $300^{\circ} \mathrm{C}$ Anal. Calcd. for $\mathrm{C}_{23} \mathrm{H}_{21} \mathrm{~N}_{5} \mathrm{O}_{3}$ (415.44): C, 66.49; H, 5.09; N, 16.86. Found: $\mathrm{C}$, 66.16; H, 4.92; N, 16.16. FTIR (KBr) $\left(\mathrm{cm}^{-1}\right): 3312,3215,3145$ $\left(\mathrm{NH}_{2}, \mathrm{NH}\right), 1679(\mathrm{CO}), 1611(\mathrm{C}=\mathrm{N}) .{ }^{1} \mathrm{H}-\mathrm{NMR}$ (DMSO-d $\left.{ }_{6}\right)$ : $\delta_{\mathrm{H}}(\mathrm{ppm}) 9.471\left(\mathrm{~s}, 1 \mathrm{H}, \mathrm{NH}, \mathrm{D}_{2} \mathrm{O}\right.$ exchangeable), 7.439-7.005 (m, 8H, Ar-H), 6.450 (s, 2H, NH $\mathrm{N}_{2}, \mathrm{D}_{2} \mathrm{O}$ exchangeable), 4.713 (s, $1 \mathrm{H}$, benzylic proton), $3.803\left(\mathrm{~s}, 3 \mathrm{H}, \mathrm{OCH}_{3}\right), 2.367(\mathrm{~s}, 3 \mathrm{H}$, $\left.\mathrm{CH}_{3}\right)$ and $1.912\left(\mathrm{~s}, 3 \mathrm{H}, \mathrm{CH}_{3}\right)$. MS, $m / z(\%): 415\left(\mathrm{M}^{\bullet+}, 10\right), 398$ (9), 372 (20), 77 (100).

2.14. Synthesis of 4-(4-Methoxyphenyl)-3,7-dimethyl-4,6-dihydropyrazolo $\left[4^{\prime}, 3^{\prime}: 5,6\right]$ pyrano $[2,3-d]$ pyrimidine-5(1H)-thione (8). A mixture of $9(10 \mathrm{mmol}, 3.4 \mathrm{~g})$ and thiourea $(10 \mathrm{mmol}$, $0.76 \mathrm{~g})$ in ethanol $(20 \mathrm{~mL})$ was heated under reflux for $6 \mathrm{~h}$. The separated solid was filtered, dried, and recrystallized from ethanol to give (8).

m.p. $218-220^{\circ} \mathrm{C}$. Anal. Calcd. for $\mathrm{C}_{17} \mathrm{H}_{16} \mathrm{~N}_{4} \mathrm{O}_{2} \mathrm{~S}$ (340): C, 59.98; H, 4.74; N, 16.46. Found: C, 60.03; H, 4.91; $\mathrm{N}$, 16.10. FTIR $(\mathrm{KBr})\left(\mathrm{cm}^{-1}\right): 3158,3121(\mathrm{NH})$, and 1225 $(\mathrm{C}=\mathrm{S}) .{ }^{1} \mathrm{H}-\mathrm{NMR}\left(\mathrm{DMSO}-d_{6}\right): \delta_{\mathrm{H}}(\mathrm{ppm}) 11.912(\mathrm{~s}, 1 \mathrm{H}, \mathrm{NH}$, $\mathrm{D}_{2} \mathrm{O}$ exchangeable), $10.112\left(\mathrm{~s}, 1 \mathrm{H}, \mathrm{NH}, \mathrm{D}_{2} \mathrm{O}\right.$ exchangeable), 7.923-7.751 ( $\mathrm{m}, 4 \mathrm{H}, \mathrm{Ar}-\mathrm{H}), 4.731$ ( $\mathrm{s}, 1 \mathrm{H}$, benzylic proton), $3.876\left(\mathrm{~s}, 3 \mathrm{H}, \mathrm{OCH}_{3}\right), 2.112\left(\mathrm{~s}, 3 \mathrm{H}, \mathrm{CH}_{3}\right)$ and $1.831\left(\mathrm{~s}, 3 \mathrm{H}, \mathrm{CH}_{3}\right)$. MS, $m / z$ (\%): $340\left(\mathrm{M}^{\bullet+}, 16\right), 281$ (19), 241 (23), 233 (100), 107 (18).

2.15. Synthesis of 5-Chloro-4-(4-methoxyphenyl)-3,7-dimethyl1,4-dihydropyrazolo $\left[4^{\prime}, 3^{\prime}: 5,6\right]$ pyrano $[2,3-d]$ pyrimidine (9). A mixture of 3 (10 mmol, $3.24 \mathrm{~g}$ ), phosphorus oxychloride $(15 \mathrm{~mL})$, and phosphorus pentachloride ( $150 \mathrm{mmol}, 3.13 \mathrm{~g})$ was heated in water bath at $80^{\circ} \mathrm{C}$ for $8 \mathrm{~h}$. The reaction mixture after cooling was poured onto crushed ice and the solid obtained was filtered off and recrystallized from ethanol to give pale brown solid (9).

m.p. $198-200^{\circ} \mathrm{C}$. Anal. Calcd. for $\mathrm{C}_{17} \mathrm{H}_{15} \mathrm{ClN}_{4} \mathrm{O}_{2}$ (342): C, 59.57; H, 4.41; N, 10.34. Found: C, 58.97; H, 3.95; N, 10.10. FTIR (KBr) $\left(\mathrm{cm}^{-1}\right): 3123(\mathrm{NH}), 1620(\mathrm{C}=\mathrm{N}){ }^{1} \mathrm{H}-\mathrm{NMR}$ $\left(\mathrm{DMSO}-d_{6}\right): \delta_{\mathrm{H}}(\mathrm{ppm}) 12.012\left(\mathrm{~s}, 1 \mathrm{H}, \mathrm{NH}, \mathrm{D}_{2} \mathrm{O}\right.$ exchangeable), $7.832-7.615$ ( $\mathrm{m}, 4 \mathrm{H}, \mathrm{Ar}-\mathrm{H}), 4.832$ ( $\mathrm{s}, 1 \mathrm{H}$, benzylic proton), $3.843\left(\mathrm{~s}, 3 \mathrm{H}, \mathrm{OCH}_{3}\right), 2.546\left(\mathrm{~s}, 3 \mathrm{H}, \mathrm{CH}_{3}\right)$ and $1.687(\mathrm{~s}, 3 \mathrm{H}$, $\left.\mathrm{CH}_{3}\right)$. MS, $m / z(\%): 342\left(\mathrm{M}^{\bullet+}, 10\right), 344(25), 235$ (12), 237 (30), 214 (28), 201 (100), 107 (30).

2.16. Synthesis of 5-Hydrazino-4-(4-methoxyphenyl)-3,7-dimethyl-1,4-dihydropyrazolo $\left[4^{\prime}, 3^{\prime}: 5,6\right]$ pyrano [2,3-d]pyrimidine (10). A mixture of 9 (10 mmol, $3.4 \mathrm{~g})$ and hydrazine hydrate $(0.5 \mathrm{~mL})$ in $(30 \mathrm{~mL})$ ethanol was heated under reflux for $5 \mathrm{~h}$. The solid separated after concentration and cooling was filtered off and recrystallized from ethanol to give (10).

m.p. $243-245^{\circ} \mathrm{C}$ Anal. Calcd. for $\mathrm{C}_{17} \mathrm{H}_{18} \mathrm{~N}_{6} \mathrm{O}_{2}$ (338.36): C, 60.34; H, 5.36; N, 24.84. Found: C, 61.07; H, 5.23; N, 24.73. FTIR $(\mathrm{KBr})\left(\mathrm{cm}^{-1}\right): 3215,3156,3161\left(\mathrm{NH}_{2}, \mathrm{NH}\right), 1612(\mathrm{C}=\mathrm{N})$ and. ${ }^{1} \mathrm{H}-\mathrm{NMR}\left(\mathrm{DMSO}-d_{6}\right): \delta_{\mathrm{H}}(\mathrm{ppm}) 12.012\left(\mathrm{~s}, 1 \mathrm{H}, \mathrm{NH}, \mathrm{D}_{2} \mathrm{O}\right.$ exchangeable), 8.123-7.836 (m, $4 \mathrm{H}, \mathrm{Ar}-\mathrm{H}), 6.013$ (s, 1H, NH, $\mathrm{D}_{2} \mathrm{O}$ exchangeable), 5.354 ( $\mathrm{s}, 2 \mathrm{H}, \mathrm{NH}_{2}, \mathrm{D}_{2} \mathrm{O}$ exchangeable), 4.803 (s, $1 \mathrm{H}$, benzylic proton), $3.734\left(\mathrm{~s}, 3 \mathrm{H}, \mathrm{OCH}_{3}\right), 2,576(\mathrm{~s}$, $\left.3 \mathrm{H}, \mathrm{CH}_{3}\right)$ and $2.087\left(\mathrm{~s}, 3 \mathrm{H}, \mathrm{CH}_{3}\right)$. MS, $m / z(\%): 338\left(\mathrm{M}^{\circ+}, 12\right)$, 307 (21), 244 (11), 201 (100), 107 (18).

2.17. Synthesis of 5-(2N,N-diacetylhydrazino)-4-(4-methoxyphenyl)-3,7-dimethyl-1,4-dihydropyrazolo $\left[4^{\prime}, 3^{\prime}: 5,6\right]$ pyrano[2, 3-d]pyrimidine (11). A mixture of $10(10 \mathrm{mmol}, 3.3 \mathrm{~g})$ was heated under reflux with $30 \mathrm{~mL}$ of fresh distilled acetic anhydride for $8 \mathrm{~h}$. The excess acetic anhydride was removed by distillation and the separated product was filtered off and recrystallized from ethanol to give (11).

m.p. 278-280 ${ }^{\circ} \mathrm{C}$ Anal. Calcd. for $\mathrm{C}_{21} \mathrm{H}_{22} \mathrm{~N}_{6} \mathrm{O}_{4}$ (422.44): C, 59.71; H, 5.25; N, 19.81. Found: C, 60.17; H, 4.96; N, 19.23. FTIR (KBr) $\left(\mathrm{cm}^{-1}\right): 3211,3198(\mathrm{NH}), 1732,1695(\mathrm{CO})$, and 1619 $(\mathrm{C}=\mathrm{N}) .{ }^{1} \mathrm{H}-\mathrm{NMR}\left(\mathrm{DMSO}-d_{6}\right): \delta_{\mathrm{H}}(\mathrm{ppm}) 12.901(\mathrm{~s}, 1 \mathrm{H}, \mathrm{NH}$, $\mathrm{D}_{2} \mathrm{O}$ exchangeable), 7.902-7.714 (m, 4H, Ar-H), $6.213(\mathrm{~s}, 1 \mathrm{H}$, $\mathrm{NH}, \mathrm{D}_{2} \mathrm{O}$ exchangeable), 5.630 ( $\mathrm{s}, 1 \mathrm{H}$, benzylic proton), 3.919 $\left(\mathrm{s}, 3 \mathrm{H}, \mathrm{OCH}_{3}\right), 2.013\left(\mathrm{~s}, 3 \mathrm{H}, \mathrm{CH}_{3}\right), 2.678\left(\mathrm{~s}, 6 \mathrm{H}, 2 \mathrm{CH}_{3}\right)$ and $1.987\left(\mathrm{~s}, 3 \mathrm{H}, \mathrm{CH}_{3}\right) .{ }^{13} \mathrm{C}-\mathrm{NMR}\left(75 \mathrm{MHz}, \mathrm{DMSO}-\mathrm{d}_{6}\right): \delta 176.6$, $174.6,173.8,171.8,163.5,160.2$, 157.2, 138.7, 130.7, 130.0, 130.0, $114.6,114.6,113.1,98.2,56.0,39.3,25.2,21.4,21.4,12.8$. MS, $m / z$ (\%): $422\left(\mathrm{M}^{\bullet+}, 13\right), 322$ (17), 307 (100), 201 (23), 107 (28), 101 (16).

\section{Conflicts of Interest}

The authors declare that they have no conflicts of interest.

\section{Acknowledgments}

The authors would like to express their appreciation for Ain Shams University.

\section{References}

[1] T. Echer and S. Hauptmann, The Chemistry of Heterocycles Structure, Reaction, Syntheses, and Applications, Wiley-VCH, Weinheim, 2003.

[2] C. G. Hartung, A. C. Backes, B. Felber, A. Missio, and A. Philipp, "Efficient microwave-assisted synthesis of highly functionalized pyrimidine derivatives," Tetrahedron, vol. 62, no. 43, pp. 1005510064, 2006

[3] C. J. Shishoo, V. S. Shirsath, I. S. Rathod, and V. D. Yande, "Design, synthesis and antihistaminic (H1) activity of some condensed 3-aminopyrimidin-4(3H)-ones," European Journal of Medicinal Chemistry, vol. 35, no. 3, pp. 351-358, 2000.

[4] R. V. Chambhare, B. G. Khadse, A. S. Bobde, and R. H. Bahekar, "Synthesis and preliminary evaluation of some $\mathrm{N}$-[5-(2-furanyl)-2-methyl-4-oxo-4H-thieno[2,3-d]pyrimidin3-yl]-carboxamide and 3-substituted-5-(2-furanyl)-2-methyl$3 \mathrm{H}$-thieno[2,3-d]pyrimidin-4-ones as antimicrobial agents," European Journal of Medicinal Chemistry, vol. 38, no. 1, pp. 89-100, 2003.

[5] M. Svobodová, P. Šimůnek, V. MacHáček, L. Štruncová, and A. Růžička, "Four-coordinate organoboron compounds from $\beta$ enaminonitriles and diazonium salts," Tetrahedron, vol. 68, no. 8, pp. 2052-2060, 2012. 
[6] D. Villemin, Z. Belhadj, N. Cheikh, N. Choukchou-Braham, N. Bar, and J. Lohier, "Solventless convenient synthesis of new cyano-2-aminopyridine derivatives from enaminonitriles," Tetrahedron Letters, vol. 54, no. 13, pp. 1664-1668, 2013.

[7] H. M. Aly and M. M. Kamal, "Efficient one-pot preparation of novel fused chromeno[2,3-d]pyrimidine and pyrano[2,3d]pyrimidine derivatives," European Journal of Medicinal Chemistry, vol. 47, no. 1, pp. 18-23, 2012.

[8] N. R. Kamdar, D. D. Haveliwala, P. T. Mistry, and S. K. Patel, "Design, synthesis and in vitro evaluation of antitubercular and antimicrobial activity of some novel pyranopyrimidines," European Journal of Medicinal Chemistry, vol. 45, no. 11, pp. 5056-5063, 2010.

[9] R. K. Rad, J. Azizian, and H. Kefayati, "Electrogenerated acetonitrile anions/tetrabutylammonium cations: an effective catalytic system for the synthesis of novel chromeno[3/,4/:5, 6]pyrano[2,3-d]pyrimidines," Tetrahedron Letters, vol. 55, no. 50, pp. 6887-6890, 2014.

[10] K. Horváti, B. Bacsa, N. Szabó et al., "Antimycobacterial activity of peptide conjugate of pyridopyrimidine derivative against Mycobacterium tuberculosis in a series of in vitro and in vivo models," Tuberculosis, vol. 95, no. 1, pp. S207-S211, 2015.

[11] F. Buron, J. Y. Mérour, M. Akssira, G. Guillaumet, and S. Routier, "Recent advances in the chemistry and biology of pyridopyrimidines," European Journal of Medicinal Chemistry, vol. 95, pp. 76-95, 2015.

[12] J. Zhang, J. Huang, C. Liu et al., "Discovery of a series of pyridopyrimidine derivatives as potential topoisomerase I inhibitors," Chinese Chemical Letters, vol. 25, no. 7, pp. 10251028, 2014.

[13] S. Labadie, K. Barrett, W. S. Blair et al., "Design and evaluation of novel 8-oxo-pyridopyrimidine Jak1/2 inhibitors," Bioorganic and Medicinal Chemistry Letters, vol. 23, no. 21, pp. 5923-5930, 2013.

[14] J. Ilaš, P. Š. Anderluh, M. S. Dolenc, and D. Kikelj, "Recent advances in the synthesis of 2H-1,4-benzoxazin-3-(4H)-ones and 3,4-dihydro-2H-1,4-benzoxazines," Tetrahedron, vol. 61, no. 31, pp. 7325-7348, 2005.

[15] D. J. Connolly, D. Cusack, T. P. O’Sullivan, and P. J. Guiry, "Synthesis of quinazolinones and quinazolines," Tetrahedron, vol. 61, no. 43, pp. 10153-10202, 2005.

[16] A. M. Alanazi, A. A. M. Abdel-Aziz, I. A. Al-Suwaidan, S. G. Abdel-Hamide, T. Z. Shawer, and A. S. El-Azab, "Design, synthesis and biological evaluation of some novel substituted quinazolines as antitumor agents," European Journal of Medicinal Chemistry, vol. 79, pp. 446-454, 2014.

[17] M. N. Noolvi, H. M. Patel, V. Bhardwaj, and A. Chauhan, "Synthesis and in vitro antitumor activity of substituted quinazoline and quinoxaline derivatives: search for anticancer agent," European Journal of Medicinal Chemistry, vol. 46, no. 6, pp. 2327-2346, 2011.

[18] A. S. El-Azab, M. A. Al-Omar, A. A. M. Abdel-Aziz et al., "Design, synthesis and biological evaluation of novel quinazoline derivatives as potential antitumor agents: molecular docking study," European Journal of Medicinal Chemistry, vol. 45, no. 9, pp. 4188-4198, 2010.

[19] K. M. Amin, M. M. Kamel, M. M. Anwar, M. Khedr, and Y. M. Syam, "Synthesis, biological evaluation and molecular docking of novel series of spiro [ $(2 \mathrm{H}, 3 \mathrm{H})$ quinazoline-2,1/- cyclohexan]$4(1 \mathrm{H})$ - one derivatives as anti-inflammatory and analgesic agents," European Journal of Medicinal Chemistry, vol. 45, no. 6, pp. 2117-2131, 2010.
[20] K. M. Pritchard, J. Al-Rawi, and C. Bradley, "Synthesis, identification and antiplatelet evaluation of 2-morpholino substituted benzoxazines," European Journal of Medicinal Chemistry, vol. 42, no. 9, pp. 1200-1210, 2007.

[21] N. Fawzy, M. O. I. Ghobashy, and A. K. El-Ziaty, "Synthesis and antimicrobial evaluation of some new quinazolin-4(3H)one derivatives," European Journal of Chemistry, vol. 3, no. 4, pp. 437-441, 2012.

[22] M. R. Mahmoud, E. A. A. El-Bordany, N. F. Hassan, and F. S. M. A. El-Azm, "New 2,3-disubstituted quinazolin-4(3H)-ones from 2-undecyl-3,1-benzoxazin- 4-one," Journal of Chemical Research, no. 9, pp. 541-544, 2007.

[23] M. El-Hashash, J. Morsy, M. Azab, and N. Mahmoud, "Design, synthesis and anticancer activity of novel 2,3-and 2,4disubstituted quinazoline and quinazolinone derivatives," Heterocycles, vol. 92, no. 2, pp. 316-329, 2016.

[24] A. M. M. El-Saghier and N. Mahmoud, "One-pot Synthesis and An Antibacterial Activity of 2-(2-hydroxyphenyl)-4oxothiazolidin-3-yl)alkyl/ phenylthiazolidin-4-one derivatives and reaction with aromatic aldehydes," Ciencia e Tecnica Vitivinicola, vol. 31, 2016.

[25] M. A. El-hashash, A. M. El-Naggar, E. A. El-Bordany, M. I. Marzouk, and T. M. S. Nawar, "Regioselectivity and regiospecificity of benzoxazinone (2-isopropyl-4H-3,1-benzoxazinone) derivatives toward nitrogen nucleophiles and evaluation of antimicrobial activity," Synthetic Communications, vol. 46, no. 14, pp. 1230-1241, 2016.

[26] M. A. El-Hashash, A. M. El-Naggar, E. A. El-Bordany, M. I. Marzouk, and T. M. S. Nawar, "6-Iodo-2-isopropyl-4H-3,1benzoxazin-4-one as building block in heterocyclic synthesis," Synthetic Communications, vol. 46, no. 24, pp. 2009-2021, 2016.

[27] H. M. F. Madkour, A. S. El-Sayed, A. I. S. Mounir, and E. A. A. El- Bordany, "Synthesis of some stable 4H-3, 1-benzoxazin4-ones and their behavior toward nucleophiles," Bulletin of the polish, vol. 47, no. 3, pp. 217-229, 1999.

[28] A. K. Elziaty, O. E. A. Mostafa, E. A. ELBordany, M. Nabil, and H. M. F. Madkour, "Access tonew pyranopyrazoles and related heterocycles," International Journal of Scientific \& Engineering Research, vol. 5, no. 1, pp. 727-735, 2014.

[29] H. Kiyani, H. A. Samimi, F. Ghorbani, and S. Esmaieli, "One-pot, four-component synthesis of pyrano[2,3-c]pyrazoles catalyzed by sodium benzoate in aqueous medium," Current Chemistry Letters, vol. 2, no. 4, pp. 197-206, 2013.

[30] M. M. Mashaly and M. Hammouda, "New simple and one-pot synthetic routes to polyfunctionally substituted pyridines; 1,4 dihydropyridazines and 4H-1,2-oxazine," Zeitschrift fur Naturforschung - Section B Journal of Chemical Sciences, vol. 54, no. 9, pp. 1205-1209, 1999.

[31] J. Gutteridge, D. Rowley, and B. Halliwell, "Superoxidedependent formation of hydroxyl radicals in the presence of iron salts. detection of free iron in biological systems by using bleomycin-dependent degradation of DNA," Biochemical Journal, vol. 199, no. 1, pp. 263-265, 1981. 

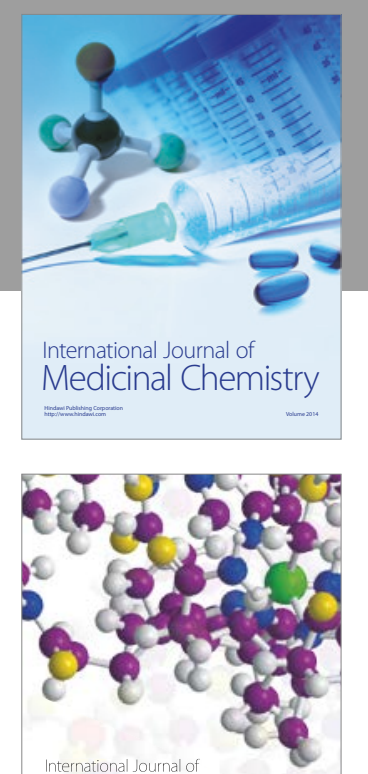

Carbohydrate Chemistry

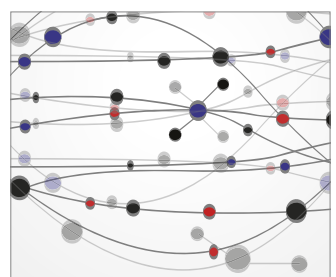

The Scientific World Journal
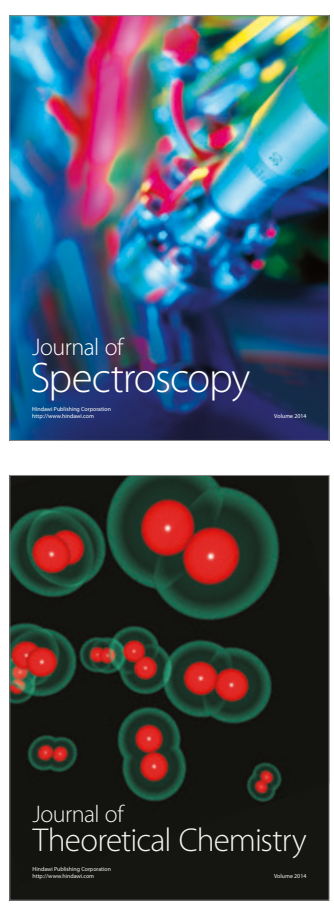
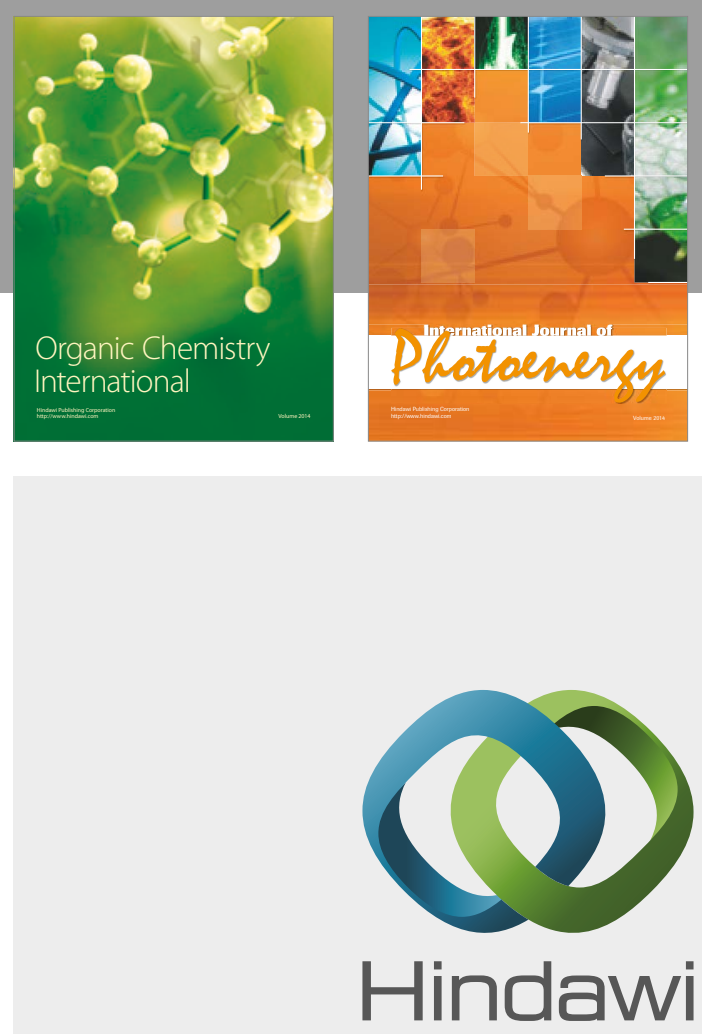

Submit your manuscripts at

https://www.hindawi.com

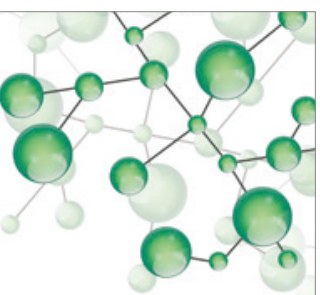

International Journal of

Inorganic Chemistry

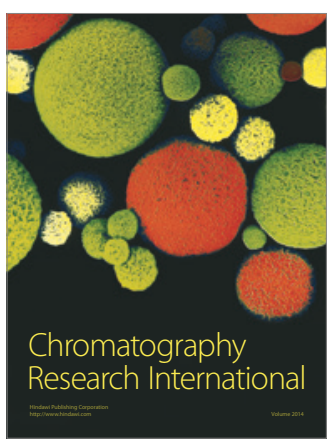

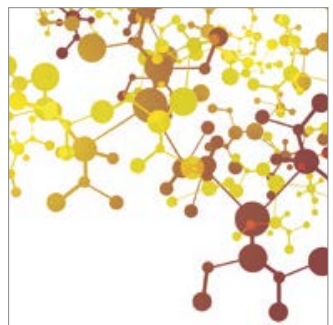

Applied Chemistry
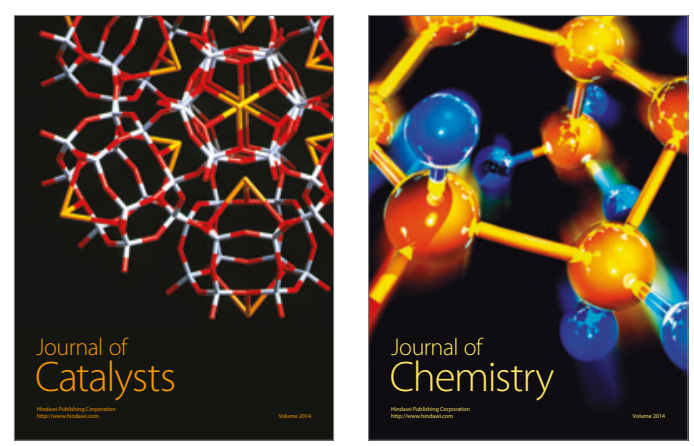
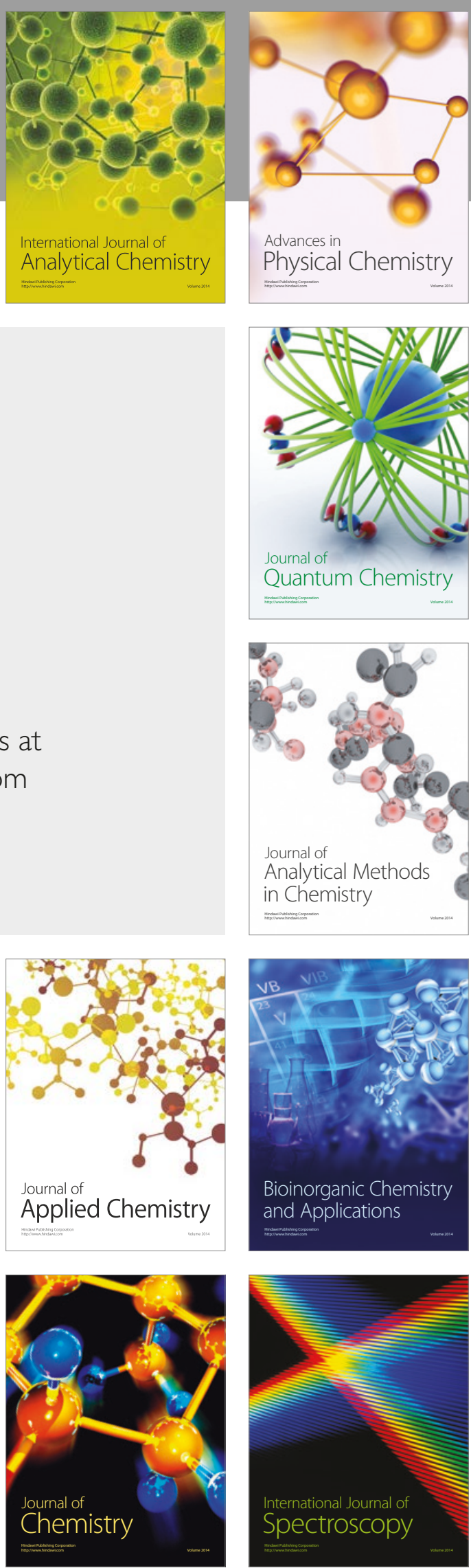